\section{Cometary organics but no evidence for bacteria}

Sir-Hoyle and N. Wickramasinghe compare the 3-4 $\mu \mathrm{m}$ spectrum of Comet Halley obtained at the Anglo-American telescope by D. Wickramasinghe and Allen ${ }^{2}$ with the predictions of a bacterial model. We believe that the agreement between the two provides no evidence for the existence of cometary bacteria.

A variety of plausible cometary materials exhibit the observed $3.4 \mu \mathrm{m}$ emission feature. These include the organic residue of irradiated candidate cometary ices ${ }^{3}$ (such as the residue of irradiated methane ice clathrate) and polycyclic aromatic hydrocarbons ${ }^{4}$, of which the residue may be partly composed. Indeed, any molecule containing $\mathrm{CH}_{3}$ and $-\mathrm{CH}_{2}-$ alkanes will emit at 3.4 $\mu \mathrm{m}$ under suitable conditions. Therefore tentative identifications must rest on additional evidence, including a plausible account of the origins of the organic material, a plausible model for the infrared emission of this material, and a demonstration that this conjunction of material and model not only matches the 3-4 $\mu \mathrm{m}$ spectrum, but also does not yield additional emission features where none is observed. In the case of the residue of irradiated low-occupancy methane ice clathrate, we have argued $^{5}$ that the synthesis of the organic residue in the laboratory well simulates the radiation processing experienced by Comet Halley, and have shown that a simple model based on the spacecraft-determined dust distribution in the Halley coma fits the 3.4 $\mu \mathrm{m}$ feature, provides optical depths in excellent agreement with those observationally determined and accounts for the absence of features at longer wavelengths. Thus any attempt to explain the spectrum of Comet Halley with living organisms or their products seems an extravagant departure from Occam's Razor.

Hoyle and Wickramasinghe neither explain nor reference their modelling procedure. The final sentence of their correspondence leaves the impression that such an account will be found in their ref. 3 , but in fact this is the paper giving the observed Halley spectrum ${ }^{2}$, which certainly reports no bacterial modelling. It is thus difficult to evaluate critically their results (shown graphically). Nevertheless a number of difficulties present themselves.

Hoyle and Wickramasinghe match the Halley data with the emission spectrum of bacteria at $320 \mathrm{~K}$. However, the continuum in the $3-4 \mu \mathrm{m}$ spectrum is best fitted ${ }^{2}$ by summing the observed flux from scattered sunlight with a black body at 350 $\pm 10 \mathrm{~K} ; 350 \mathrm{~K}$, adjusted for heliocentric distance, is also the continuum temperature found by Danks et al. ${ }^{6}$ in the 3-4 $\mu \mathrm{m}$ range, as well as (to within the $10 \mathrm{~K}$ uncer- tainty) that which best fits the $5-13 \mu \mathrm{m}$ continuum ${ }^{7}$. Thus the temperature used by Hoyle and Wickramasinghe lies outside the observational error bars. Moreover, the feature at $\sim 3 \mu \mathrm{m}$, which is somehow well-fitted by the bacterial emission spectrum, is revealed by both ground-based observations ${ }^{2}$ and those made by the Vega spacecraft ${ }^{8}$ to be an absorption feature (probably the $\mathrm{O}-\mathrm{H}$ vibrational transition) lying well below the continuum flux.

The choice of a single temperature to characterize both the continuum and the emission feature, as well as the description of their model curve as simply "the predicted spectrum of bacteria heated to 320 K", indicates that Hoyle and Wickramasinghe are in effect treating the entire 3-4 $\mu \mathrm{m}$ spectrum (both emission feature and continuum) as due to emission from bacteria. However, there are grave objections to such a procedure. The intensity of the $3.4 \mu \mathrm{m}$ band varies independently of the continuum by as much as a factor of 4.5 , suggesting that the continuum and emission feature are produced by two independent dust components ${ }^{2}$ (indeed, this is also the interpretation given to the two-fold variation of the $10 \mu \mathrm{m}$ silicate dust feature relative to the continuum $)^{7}$. The continuum flux must be due primarily to the $\sim 98 \%$ of the coma dust-filling factor provided by grains $\geqslant 1.0 \mu \mathrm{m}$ in radius. ${ }^{5}$ Grains with radii $\geqslant 10 \mu \mathrm{m}$, comprising $\sim 77 \%$ of the coma-filling factor, will emit as black bodies (being individually optically thick at 3-4 $\mu \mathrm{m}$ ); the bacterial spectrum is in any case irrelevant for these. The $3.4 \mu \mathrm{m}$ emission feature, on the other hand, must be due to grains that are individually optically thin at this wavelength, that is the $\sim 2 \%$ of the dust-filling factor due to grains with radii $\leqslant 1.0 \mu \mathrm{m}$. Hanner ${ }^{9}$ has demonstrated that such submicron cometary dust will have temperatures hundreds $\mathrm{K}$ hotter than a black body at $1 \mathrm{AU}$. Thus an appropriate temperature for the submicron emitting grains is probably $\sim 500 \mathrm{~K}$, not $320 \mathrm{~K}$.

Both spacecraft and ground-based near-infrared observations, as well as the results of in situ mass spectroscopy ${ }^{10}$, strongly suggest the presence of complex organic or organic-coated grains in the Halley coma. It would be unfortunate if claims for living organisms prejudiced the reception of these important results.

\section{Christopher Chyba}

Laboratory for Planetary Studies,

Cornell University,

Ithica, New York 14853, USA

1. Hoyle. F. \& Wickramasinghe, N.C. Nature 328, 117 (1987).

2. Wickramasinghe, D.T. \& Allen, D.A. Nature 323, 44-46 (1986)

Khare, B.N. et al. Icarus (in the press)

4. Knacke, R.F. et al. in 20 th Eslab Symposium on the Explor ation of Halley's Comet 2, 95-100 (ESA SP - 250, 1986). Chyba, C. \& Sagan, C. Nature (in the press)

5. Chyba, C. \&agan, C. Nature (in the press)

Danks, A. et al. in 20trh Eslab Symposium on the Explor ation of Halley's Comet 3, 103 - 106 (ESA SP-250, 1986). Bregman, J.D. et al. Astr. Astrophys. (in the press)

. Combes, M. et al. Adv. Space Sci. (in the press).

9. Hanner. M.S. in Cometary Exploration II (cd. Gombosi T. I, 1-22 (Hungarian Acad. Sci. 1982)

10. Kissel. J. \& Krueger. F. R. Nature 326. 755-760)(1987)

\section{The blood - brain barrier}

SIR-The endothelium of cerebral blood capillaries forms a cellular barrier with selective properties that isolates circulating compounds in the blood from those produced by the brain, so maintaining the homoeostatic environment of the brain ${ }^{1.2}$. The perivascular glial cells may play a role in the induction and maintenance of these barrier properties.

In elegant experiments, Janzer and Raff ${ }^{3}$ recently provided clear evidence for the primary role of type I astrocytes in the induction of impermeability to macromolecules in the capillaries of the iris. The finding raises several intriguing questions including the nature of the inducing signal and the importance of cell-to-cell communication. I think, however, that there is an important reservation about the interpretation of these experiments, as Brightman and Reese ${ }^{4}$ and Reese and Karnovsky ${ }^{5}$ have shown that the cerebral endothelium differs in structure from that of peripheral vessels in two ways: first, in cerebral vessels there are highly impermeable tight junctions between endothelial cells, and second, the cells have much lower pinocytotic activity than peripheral endothelial cells. It is these two features that give the cerebral endothelium its selective properties. Vasoactive substances, such as histamine can activate pinocytosis ${ }^{6}$ without opening the tight junctions ${ }^{7}$ indicating that activation of pinocytosis alone is sufficient to change the permeability of the barrier. The low rate of pinocytosis may also contribute to the impermeability of these vessels to macromolecules .

The model of Janzer and Raff will certainly be useful for further studies of the transformation of a peripheral endothelium into one with features of cerebral capillaries. I consider, however, that fine structural investigations are essential to determine whether the endothelial cells have cerebral or peripheral characteristics. Without this information it is not possible to tell with certainty which of the structural features determines development of impermeability to macromolecules. But it would not surprise me if the suppression of pinocytosis turns out to be the worthy side of the coin.

FERENC Joó

National Institute of Neurological and

Communicative Disorders and Stroke, National Institutes of Health, Bethesda, Maryland 20892, USA

Bradbury, M. Concept of a Blood-Brain Barrier (Wiley, Chichester, 1979).

2. Cserr, H.F. \& Bundgaard, M Am. J. Physiol. 246, R277 (1984)

3. Janzer, R.C. \& Raff, M.C. Nature, 325, 253 (1987).

4. Brightman, M.W. \& Reese, T.S. J. Cell Biol. 40, 648 (1969).

. Reese, T.J. \& Karnovsky, M.J. J. Cell Biol. 34.207 (1967). 6. Dux, E. \& Joó, F. Expl brain Res. 47, 252 (1982)

Dux, E. et al. Neuroscience, 12, 951 (1984)

8. Joó. F. Nature 321, 197 (1986) 